\title{
Nonradial adiabatic oscillations of stars
}

\section{Mode classification of acoustic-gravity waves}

\author{
I. P. Lopes ${ }^{1,2}$ \\ 1 Department of Physics, Nuclear and Astrophysics Laboratory, Keble Road, Oxford OX1 3RH, UK \\ 2 Instituto Superior Técnico, Av. Rovisco Pais, Centro Multidisciplinar de Astrofísica, 1049-001 Lisboa, Portugal
}

Received 31 May 2000 /Accepted 17 January 2001

\begin{abstract}
The classification scheme of mode oscillations in stars is investigated mathematically and physically by using a new phase diagram representation. This technique is presented in order to study the basic equation of motion for linear adiabatic nonradial oscillations, and leads us to obtain an unambiguous scheme classification of modes. The order number introduced by this classification scheme only depends on the boundary conditions at the endpoints, the centre and the surface of the star. Furthermore, this classification is independent of the number of nodes of the eigenfunction used to characterize the oscillatory motion. Consequently, the sequence of ordinate eigenfrequencies becomes the signature of the oscillatory system itself. Provided that the equation of motion of linear adiabatic nonradial stellar oscillations has been reduced to a second-order differential equation, this technique allows us to obtain a more reliable classification scheme of modes in stars. Although this phase analysis method has been presented to characterize the propagation of acoustic-gravity waves with one or two propagative regions, it can be nevertheless successfully applied to perturbative motions with more than three propagative regions, provided that a propagation diagram can be built.
\end{abstract}

Key words. stars: oscillations - stars: interiors - Sun: oscillations - Sun: interior

\section{Introduction}

The progress of stellar seismology is strongly dependent on our understanding of the basic properties of the stationary waves in the interior of the star. In particular, one of the cornerstones of theoretical stellar seismology is the determination of a correct classification scheme of the eigenmodes that can be present for a given equilibrium structure. Usually there are two ways of investigating the classification scheme of stellar nonradial oscillations, where one complements the other. The numerical method is used to calculate the lower overtones and the asymptotic methods (Shibahashi 1979; Tassoul 1980; Gough 1996; Provost \& Berthomieu 1986; Smeyers \& Tassoul 1988; Tassoul 1990; Vorontsov 1991; Gough 1993) are applicable to the higher overtones. This formal mathematical classification is crucial to progress in the development of the analytical dispersion relation of stationary waves (Gough 1993), and in the improvement of the inversion methods.

Cowling (1941) was the first to introduce a classification scheme for waves, based on the two restoring forces present inside a star in hydrostatic equilibrium, without magnetics fields or differential rotation: the pres-

Send offprint requests to: I. P. Lopes

e-mail: lopes@astro.ox.ac.uk sure and the gravity. The Cowling classification scheme divides stationary waves into three types: the gravity modes ( $g$-modes $)$, the acoustic modes $(p-$ modes $)$ and the f-mode (Deubner \& Gough 1984; Gough 1993; Gough 1996; Unno et al. 1989). This classification is based on the local properties of the waves which attribute to each mode a order number determined by the number of nodes of the radial eigenfunction. In particular, the $f$-mode is a mode with a radial eigenfunction with no nodes. It is easy to classify nonradial oscillations for simple stellar equilibrium structures, as the less condensed polytropes or even the zero-age main-sequence stars. This is because the propagation zones for each restoring force are clearly separated and they can be seen as a simple generalization of the Sturm-Liouville type problem ${ }^{1}$. For more evolved stars, the propagation zones of acoustic and

\footnotetext{
1 Strictly speaking, this is not a Sturm-Liouville problem because the dependence of the eigenvalue with the equilibrium model is rather complicated. However, the main properties of this oscillatory system are quite similar to the classical SturmLiouville problem. This has been put in evidence by Ledoux \& Walraven (1958) for the high order gravity modes and the high order acoustic modes, where a second-order system of stellar oscillations has been obtained under the Cowling approximation (see Sect. 3.4).
} 
gravity waves overlap with each other. In some cases, there are even more complicated propagation diagrams with three or more propagation zones for a given wave, as for evolved stars of $10 M_{\odot}$ (Unno et al. 1989). In such cases, the classification of nonradial modes is not trivial. Different generalizations have been made by Scuflaire (1974) and Osaki (1975), where the Cowling scheme has been generalized. However, some problems have still remained in these new schemes and the determination of an unambiguous classification method has not yet been achieved. More recently, in a tentative to obtain a scheme that better represents the properties of modes, Shibahashi \& Osaki (1976a, 1976b) introduced a classification scheme based on the behaviour of the modes in the trapping zone. These classification schemes present two main problems that difficult their use as a general classification procedure. First, the fourth-order system of nonradial adiabatic stellar oscillations has been reduced to a second-order system by approximative methods, which makes impossible to obtain a general scheme classification for the complete full problem. Second, the classification scheme is dependent of the eigenfunction that has been chosen to characterize the oscillatory motion because the order of the mode is determined by counting the number of nodes of this radial eigenfunction (Cox 1980). The classification scheme presented in this article will try to overcome this second problem.

Our goal in this article is to present a classification scheme, for which the number associated to the order of the modes is independent of the eigenfunction chosen to characterize the oscillatory motion. We will analyse with particular interest the low overtones which present some peculiar behaviour for some complicated stellar structure models. The method presented here is a particular phase representation that is made for the second-order differential equation, written in an appropriate self-adjoint form. This new method is a generalization of the method developed by Prüfer (1926), to study the classical SturmLiouville problem. The technique presented can be successfully applied for all the overtones from the smaller to the higher ones. An analogous procedure has already been used by Gabriel \& Scuflaire (1979). This type classification scheme as also being study by Lee (1985) in the specific case of dipole modes. Lopes \& Gough (2001) and Lopes et al. (1997) as also used this technique to determine the phase shift produced in the outer layers of the Sun and solar-like stars by the partial ionization of hydrogen and helium. A new variant of this method is used to calculate the eigenfrequency equation of low degree modes of acoustic oscillations (Lopes 2001).

The next section contains a brief presentation of the motion equation of stellar oscillations for a spherically symmetrical background state, where some approximative results are mentioned. This is followed by the presentation of the new phase method to study the second-order equation of oscillatory motion, where the mathematical and physical properties of the acoustic-gravity waves are discussed. In Sect. 5, we present the new classification scheme, where we discuss the relation between this new scheme and the ones currently used. In the last section, a summary and conclusion of the main results here established are presented and we also point out the usefulness of the results obtained for the general study of stellar oscillations.

\section{Basic equation of oscillatory motion}

Formally, the nonradial adiabatic oscillations of stars can be described as the solution of the simple linear homogeneous adiabatic wave equation:

$\square \Psi=\left[\frac{\partial^{2}}{\partial t^{2}}+\mathcal{L}\right] \Psi=0$

where $\square$ is the adiabatic wave operator and $\Psi$ is some wave function depending on the position vector $\boldsymbol{r}$ and the time $t$, that characterizes the oscillations (Gough 1993, 1996). The oscillatory motion under study is supposed to be of such low amplitude that linearization of the full equations of fluid dynamics is valid, allowing to determine the wave equation that describes the oscillatory movements.

Therefore, we are ignoring the interactions between waves, non-adiabatic effects which are important only in beneath the atmosphere, and effects of the turbulence in the convective zone. We also consider a non-magnetic and non-rotating star. The adiabatic wave operator depends on the structure of the solar model, which we will refer to as the background state. We have assumed that a frame of reference exists in which the background state is independent of time. In this case, there are genuinely separable solutions $\Psi(\boldsymbol{r}, t)=\mathcal{R}\left[\Psi(\boldsymbol{r}) \mathrm{e}^{-i \omega t}\right]$ of the simple homogeneous adiabatic equation. Considering that the wave has a pure dependence on $t$ with frequency $\omega$, the spatial part of the wave function, $\Psi(\boldsymbol{r})$ satisfies:

$\mathcal{L}_{\omega} \Psi=0$

where the three-dimensional spatial differential operator $\mathcal{L}_{\omega}$ is obtained from the full wave operator $\square$ by replacing $\frac{\partial}{\partial t}$ by $-i \omega$. We will use $\Psi_{r}^{2}$ to represent the factor depending on $r$ in the separated form $\Psi(\boldsymbol{r})=\Psi_{r}(r) Y_{l}^{m}(\theta, \phi)$ with respect to the spherical polar coordinates $(r, \theta, \phi)$ when the background state is spherically symmetric, and where $Y_{l}^{m}(\theta, \phi)=P_{l}^{m}(\cos \theta) \mathrm{e}^{i m \phi}$ is a spherical harmonic of degree $l$ and azimuthal order $m, P_{l}^{m}$ being the associated Legendre function of first kind. In this case, the separation of variables into radial and angular parts is possible for all background variables, with the angular dependence of $Y_{l}^{m}(\theta, \phi)$ satisfying the eigenvalue equation,

$r^{2} \nabla_{\perp}^{2} Y_{l}^{m}(\theta, \phi)=-L^{2} Y_{l}^{m}(\theta, \phi)$

where $\nabla_{\perp}$ is the horizontal Laplace operator, $m=$ $-l, \ldots,+l$ and $L^{2}=l(l+1)$. In that case $\mathcal{L}_{\omega}$ will represent the radial part of the corresponding three-dimensional operator with the same name. In the spherically symmetric

\footnotetext{
${ }^{2}$ Hereafter we will refer to $\Psi$ as $\Psi_{r}$, if not stated otherwise.
} 
case, the solutions of Eq. (2) together with appropriate boundary conditions, admit discrete eigenfrequencies $\omega_{n l}$, where $n$ is the radial wave number. The spherical symmetry of the background state is responsable by the degeneracy of $\omega_{n l}$ with respect to $m$.

In the case of a spherically symmetrical background state, the homogeneous differential equation (Eq. (2)) representing adiabatic oscillations is of fourth-order and has to be solved subject to two regularity conditions at the coordinate singularity $r=0$, and to two boundary conditions at the surface $r=R$ (Unno et al. 1989; Gough 1993).

Taking into account the mathematical structure of the operator $\mathcal{L}_{\omega}$, it is possible to reduce this one to the standard second-order differential equation. This equation of motion can be obtained directly from a linearized Eulerian momentum equation and from the Poisson equation that describes the oscillatory motion by making a convenient transformation (Gough 1993). In the following, we make a very brief presentation of approximations to the secondorder motion equations.

\subsection{The standard form}

The linear adiabatic nonradial stellar oscillations of a spherically symmetrical background state can be written in a standard form. Generically we can write the motion equation of adiabatic nonradial oscillations as

$$
\frac{\mathrm{d}^{2} \Psi}{\mathrm{d} r^{2}}+k_{\mathrm{r}}^{2} \Psi=0
$$

where the radial component of the local wave number, $k_{\mathrm{r}}$, is given by

$$
k_{\mathrm{r}}^{2}=\frac{\omega^{2}-\omega_{\mathrm{c}}^{2}}{c^{2}}-\frac{L^{2}}{r^{2}}\left(1-\frac{\mathcal{N}^{2}}{\omega^{2}}\right)
$$

where $c$, is the radial distribution of sound speed, $\omega_{\mathrm{c}}$, defines a generalized critical frequency and $\mathcal{N}$, a generalized Brunt-Väisälä frequency, that takes into account the geometrical terms and gravity contribution for the local wave number, which will be defined for each of the approximative motion equations. $\Psi$ is the wave function, which is proportional to the Lagrangian perturbation of pressure and will take a particular dependence, in agreement with the approximation used.

In the following analysis we will always consider this general case given by Eq. (4), unless we say explicitly the contrary.

\subsubsection{The Planar approximation}

In 1984, Deubner \& Gough determined a linear secondorder differential equation in the standard form of the Eq. (4), to describe the dynamics of adiabatic non-radial oscillations. This equation of motion has been obtained by a procedure analogous to the Lamb (1932) method. This approximation can be made for waves with wavelength much smaller than the solar radius and where the local effects of spherical geometry on the oscillatory motion can be ignored. Additionally, the perturbations of the gravitational potential have been ignored. In this case, the wave function is given by,

$\delta p=\rho^{1 / 2} \Psi$

where $\rho$, is the density and $\delta p$, is the Lagrangian perturbation of pressure. In this case, the local wave number, $k_{\mathrm{r}}$, is given by Eq. (5), where the critical frequency, $\omega_{\mathrm{c}}$, is given by

$\omega_{\mathrm{c}}^{2}=\frac{c^{2}}{4} \frac{1}{H^{2}}\left(1-2 \frac{\mathrm{d} H}{\mathrm{~d} r}\right)$,

where $H$, is the density scale height. The generalized Brunt-Väisälä frequency, $\mathcal{N}$, is reduced to the classical Brunt-Väisälä frequency, $N$, given by

$N^{2}=g\left(H^{-1}-\frac{g}{c^{2}}\right)$

where $g$, is the gravitational acceleration. This model is particularly interesting because of the simplicity in the interpretation of the characteristic frequencies of the equilibrium structure that determine the cavities of propagative waves. All the main properties of the local behavior of waves, can be well defined in this approximation.

\subsubsection{The Cowling approximation}

The equation of motion of stellar oscillations can be obtained by reducing the fourth-order system of stellar oscillations to a second-order differential equation, where the Eulerian perturbation of the gravitational potential is neglected (Gough 1993). Cowling (1941) showed the interest of this approximation, by pointing out that it has a relatively minor effect on the modes, with exception to the modes of low degree and low order. Under the approximation proposed, i.e., neglecting the contribution of the Eulerian pertubation of gravitational potential, $\Phi^{\prime}$, the initial fourth-order system of adiabatic stellar oscillations is reduced to a second-order motion equation, by the following transformation

$\delta p=\operatorname{sgn}(f)\left|\frac{g \rho f}{r^{3}}\right|^{1 / 2} \Psi$,

where $\operatorname{sgn}(f)$, is the sign of the discriminant $f$. This discriminant, is given by

$f=4+\left(\omega^{2}-\omega_{J}^{2}\right) \frac{r}{g}-\frac{L^{2}}{\omega^{2}} \frac{g}{r}$.

$\omega_{\mathrm{J}}^{2}$, is the square of the Jeans frequency, that is equal to $4 \pi G \rho$, where $G$ is the gravitational constant. Using this transformation, the motion equation of adiabatic nonradial stellar oscillations, can be written in the form of Eq. (4). In that case, the generalized Brunt-Väisälä frequency, $\mathcal{N}$, is given by

$\mathcal{N}^{2}=g\left(\mathcal{H}^{-1}-\frac{g}{c^{2}}-2 h^{-1}\right)$ 
and the critical frequency, $\omega_{\mathrm{c}}$, is given by

$\omega_{\mathrm{c}}^{2}=\frac{c^{2}}{4}\left(\mathcal{H}^{-2}+2 \frac{\mathrm{d} \mathcal{H}^{-1}}{\mathrm{~d} r}\right)-\frac{g}{h}$,

where $h$ is the scale height of $g / r^{2}$ and $\mathcal{H}$ is a generalized scale height. The scale height $h$, is given by

$h^{-1}=4 r^{-1}-\frac{\omega_{\mathrm{J}}^{2}}{g}$

and $\mathcal{H}$, is given by

$\mathcal{H}^{-1}=3 r^{-1}+H^{-1}+H_{\mathrm{g}}^{-1}+H_{f}^{-1}$,

where $H_{\mathrm{g}}$, is the gravity scale height and $H_{f}$, is the scale height of discriminant $f$. A detailed discussion about these variables is presented in Gough (1993).

The difference between the generalization of the BruntVäisälä frequency, $\mathcal{N}$ and the critical frequency, $\omega_{c}$ relatively to these quantities in the Deubner \& Gough (1984) approach, is due to the contribution of geometrical terms, that can be important for the most penetrative modes in very dense stars.

\subsubsection{The first post-Cowling approximation}

A more general solution to the initial system of stellar oscillations can be obtained, which takes into account a major contribution related with $\Phi^{\prime}$. This is called the first Post-Cowling approximation (Gough 1993; Dziembowski \& Gough, private comunication). Under this approximation the Brunt-Väisälä frequency, $\mathcal{N}$, is given by

$\mathcal{N}^{2}=g\left(\frac{1}{\mathcal{H}}-\frac{g}{c^{2}}-\frac{2}{h}\right)$

and the critical frequency, $\omega_{c}$, is given by

$\omega_{\mathrm{c}}^{2}=\frac{c^{2}}{4 \mathcal{H}^{2}}\left(1-2 \frac{\mathrm{d} \mathcal{H}}{\mathrm{d} r}\right)-\frac{g}{h}-\omega_{\mathrm{J}}^{2}$.

The total scale height $\mathcal{H}$, is given by

$\mathcal{H}^{-1}=3 r^{-1}+H^{-1}+H_{\mathrm{g}}^{-1}+H_{f}^{-1}+H_{\varphi}^{-1}$,

where the scale height $H_{\varphi}$ takes into account the contribution related with the perturbation of the gravitational potential (Gough 1993; Dziembowski \& Gough, private comunication; Lopes 2001). The scale height $H_{\varphi}$, is given by

$H_{\varphi}^{-1}=\frac{\omega_{\mathrm{J}}^{2} r}{g f H}$

The wave function, $\delta p$, can be determined as

$\delta p=\operatorname{sgn}(f)\left|\frac{g \rho f \varphi}{r^{3}}\right|^{1 / 2} \Psi$

where $\varphi$, is a structure term related with the background state, given by

$\varphi(r)=\int_{0}^{r} H_{\varphi}^{-1} \mathrm{~d} r$.
These new expressions for the characteristic frequencies come from a contribution related with the perturbation of the gravitational potential which can be obtained from the linearized Poisson equation. The magnitude of the effect relatively to the classical Cowling approximation strongly depends on the density distribution in the central region of the star.

\subsection{Boundary conditions}

The second-order motion equation together with two boundary conditions, one at the centre and the other at the surface, forms an eigen-value problem. We will present these boundary conditions here, as they will be needed later.

The center is a regular singular point. The regularity condition for the perturbation of the Lagrangian variation of pressure, $\delta p$, is given by

$\frac{\mathrm{d} \delta p}{\mathrm{~d} r}-\frac{l}{r} \delta p \rightarrow 0$

as $r \rightarrow 0$. The outer boundary condition, is fixed in the atmosphere and we consider that the inertia of the corona is so high that $\delta p$, is given approximately by

$\delta p=0$

at $r=R$. These simple conditions are sufficient to the discussion proposed in this article. These are the socalled "zero-boundary conditions" (Unno et al. 1989). The consideration of more realistic boundary conditions does not modify significantly the method proposed to classify modes in stars.

\subsection{Propagation diagram}

The direction and magnitude of the acoustic-gravity wave is determined by the competition between the radial and tangential components of the local wave number $\boldsymbol{k}$, given by

$\boldsymbol{k}=k_{\mathrm{r}} \boldsymbol{e}_{\mathrm{r}}+k_{\mathrm{h}} \boldsymbol{e}_{\mathrm{h}}$,

where $\boldsymbol{e}_{\mathrm{r}}, \boldsymbol{e}_{\mathrm{h}}$ are unitary vectors in the radial and horizontal directions and $k_{\mathrm{h}}\left(\equiv L^{2} / r^{2}\right)$, is the tangential component of the local wave number (Unno et al. 1989). The radial component of the local wave number, is determined by the local properties of the equilibrium structure. In this sense, it is possible to build a diagram for the radial component of the local wave number, which defines the regions of propagative or evanescent behavior of a given acoustic-gravity wave of frequency $\omega$ and degree $l$. This is the so-called propagation diagram. Waves can propagate where the square of the vertical component of the local wave $k_{\mathrm{r}}$ is greater than zero, and are evanescent elsewhere. This leads to two asymptotic limits, for the very high and for the very small frequencies. In the case of very high frequencies, $k_{\mathrm{r}} \gg k_{\mathrm{h}}$, the propagation is predominantly in the radial direction. In the other case, $k_{\mathrm{r}} \ll k_{\mathrm{h}}$, then 

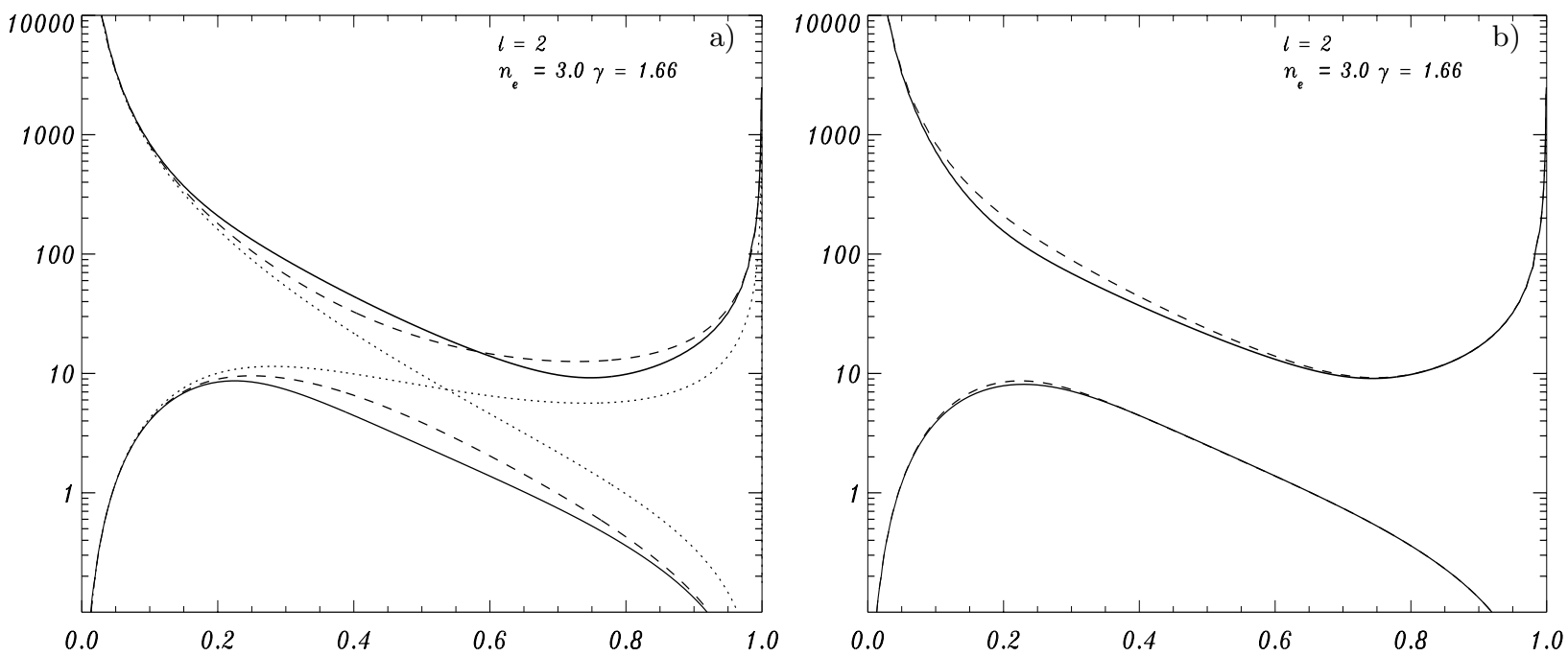

Fig. 1. Propagation diagram for a polytropic model with index $n_{\mathrm{e}}=3.0$, adiabatic index $\gamma=5 / 3$. The curves correspond to a dimensionless circular frequency relatively to the dimensionless radius. These curves delimit the propagation region for modes of degree $\ell=2$ : a) The solid curves represent $\omega_{ \pm}^{2}$ computed in the Cowling approximation, the dashed curves represent planar values of $\omega_{ \pm}^{2}$, and the dotted curves represent the Brünt-Väisäla frequency, $N$, (increasing from the center to the surface) and the Lamb frequency, $S_{\ell}$, (decreasing from the center to the surface). b) The solid curves represent $\omega_{ \pm}^{2}$ computed in the first Post-Cowling approximation, and the dashed curve represents $\omega_{ \pm}^{2}$ in the Cowling approximation.

the propagation is predominantly in the horizontal direction. This corresponds also to the spectral regions where the restoring forces are dominated by gravity (through the buoyancy) or pressure. Because of that, it is convenient to write the local wave number as a function of the critical frequencies $\omega_{ \pm}^{2}$, in which case $k_{\mathrm{r}}$, is given by

$k_{\mathrm{r}}^{2}=f_{\mathrm{g}} f_{\mathrm{p}}$

where $f_{\mathrm{g}}$ is a discriminant, given by

$f_{\mathrm{g}}=\frac{\omega}{c}\left(1-\frac{\omega_{-}^{2}}{\omega^{2}}\right)$

and $f_{\mathrm{p}}$ is a discriminant, given by

$f_{\mathrm{p}}=\frac{\omega}{c}\left(1-\frac{\omega_{+}^{2}}{\omega^{2}}\right)$.

The underscript $\mathrm{g}$ or $\mathrm{p}$ is fixed by the propagative regions that are separated by $\omega_{-}$or $\omega_{+}$, respectively.

It follows from Eq. (23) that $k_{\mathrm{r}}^{2}>0, \omega_{ \pm}^{2}$ are real and $f_{\mathrm{p}}>0$ and $f_{\mathrm{g}}>0$ (acoustic or p-region, locally behaves like an acoustic type wave) or $f_{\mathrm{p}}<0$ and $f_{\mathrm{g}}<0$ (gravity or $g$-region, locally behaves like a gravity type wave). Here we will be mainly concerned with the case where $\omega_{+}^{2}$ and $\omega_{-}^{2}$ are both real. In the case where $\omega_{-}^{2}$ and $\omega_{+}^{2}$ are complex conjugates $^{3}$ it is also possible to use the same analysis. It will slightly complicate the algebra, but the physical conclusions remain the same.

The critical frequencies $\omega_{ \pm}^{2}$, define the turning points of the standard second-order differential equation, previously presented, i.e., $k_{\mathrm{r}}^{2}=0$. In the more general case,

\footnotetext{
${ }^{3}$ We observe that if $k_{\mathrm{r}}^{2}$ is real, then $\omega_{-}^{2}$ and $\omega_{+}^{2}$ are both real or complex conjugates. Consequently $f_{\mathrm{p}}$ and $f_{\mathrm{g}}$ are also complex conjugate functions.
}

$\omega_{ \pm}^{2}$, can be determined numerically. However the critical frequencies $\omega_{ \pm}^{2}(\omega, l ; r)$ can be obtained, by the relation,

$$
\omega_{ \pm}^{2}(\omega, l ; r)=\frac{1}{2}\left(S_{\mathrm{l}}^{2}+\omega_{\mathrm{c}}^{2}\right) \pm \sqrt{\frac{1}{4}\left(S_{\mathrm{l}}^{2}+\omega_{\mathrm{c}}^{2}\right)^{2}-\mathcal{N}^{2} S_{\mathrm{l}}^{2}}
$$

where, the Lamb frequency $S_{\mathrm{l}}$, is given by

$S_{1}^{2}=l(l+1) \frac{c^{2}}{r^{2}}$

In the case of Deubner \& Gough (1984), these expressions determine an explicit relation of the critical frequencies as function of the background structure and independent of the frequency (see Sect. 2.1.1). In Figs. 1 and 2 we present the computed critical frequencies, $\omega_{ \pm}^{2}$, for the different approximations presented in the previous section, in the case of polytropes of index $n_{\mathrm{e}}=3$ and $n_{\mathrm{e}}=4$ and adiabatic in$\operatorname{dex} \gamma=5 / 3$. A discussion about the propagation of waves in polytropic equilibrium structure with a different adiabatic index can be found in Cowling (1941) and Scuflaire (1974). The contribution related with the geometric terms occuring in the variables $\mathcal{N}$ and $\omega_{\mathrm{c}}$ when computed in the Cowling approximation is more important for the more penetrative waves, i.e., waves with smaller degree, as it is illustrated in Figs. 1a and 2a. Similarly, the first PostCowling approximation, seems worth considering in the case of stars with high density, where the presence of the gravitational potential modifies significantly the values of $\omega_{ \pm}^{2}$ in the central region of the star (cf. Figs. $1 b$ and $2 b$ ).

In the next section, using the differential Eq. (4) with the local wave number given by Eq. (23), we will write the standard equation of motion in a convenient self-adjoint form, very appropriate for the phase analysis that we will present below. 


\section{Phase analysis representation of the equation of motion}

The phase method proposed here is based in a generalization of a technique normally used to study the solutions of a particular type of self-adjoint second-order linear differential equation with homogeneous boundary conditions, the so-called Sturm-Liouville problem. This method consists in representing the eigenmodes in a convenient Poincaré phase plane (in terms of polar coordinates), normally used to study an autonomous system of differential equations.

In a first step, we present two complementary selfadjoint forms of the standard second-order differential equation that describes the adiabatic nonradial oscillations of a spherically symmetric star (Eq. (4)). Second, we present the phase analysis method to determine the eigenstates of each of these self-adjoint differential equations, where we introduce a classification scheme that is build using the boundary conditions.

\subsection{Complementary self-adjoint differential equations}

The standard second-order differential equation of linear adiabatic stellar oscillations (Eq. (4)), can be written into a pair of self-adjoint differential equations. As we will discuss later, these equations should then be regarded as being complementary to each other, for the study of the local properties, related with the two driving mechanisms, pressure and gravity. The transformation of the second-order differential equation into a self-adjoint form will be done, taking into account the previous decomposition related with the two propagation cavities for a given wave. This is accomplished by the transformation,

$\Psi=\mathcal{M}_{i} \mathcal{Y}_{i}$,

where $i=1,2$ defines the two possible transformations, related with two propagative regions. We point out that, in the most general case, under these transformations $\mathcal{Y}_{i}$ does not conserve the location and the number of zeros of the wave function $\Psi$. We consider also that $\mathcal{M}_{i}$ is a real function. However, if the function $\mathcal{M}_{i}$, is conveniently chosen (with no zeros in the whole interval considered) it is possible to determine the location of the zeros for the wave function $\Psi$, by the location of zeros of $\mathcal{Y}_{i}$. Resulting in the following self-adjoint equation of motion,

$\frac{\mathrm{d}}{\mathrm{d} r}\left[\mathcal{P}_{i}(r) \frac{\mathrm{d} \mathcal{Y}_{i}}{\mathrm{~d} r}\right]+\mathcal{Q}_{i}(r) \mathcal{Y}_{i}=0$,

where $\mathcal{P}_{i}$, is given by

$\mathcal{P}_{i}(r)=\operatorname{sgn}\left(\mathcal{M}_{i}\right)\left|\mathcal{M}_{i}\right|^{2}$

and $\mathcal{Q}_{i}$, is given by

$\mathcal{Q}_{i}(r)=\mathcal{P}_{i}\left(k_{\mathrm{r}}^{2}+\frac{1}{\mathcal{M}_{i}} \frac{\mathrm{d}^{2} \mathcal{M}_{i}}{\mathrm{~d} r^{2}}\right)$.

The function $\mathcal{M}_{i}$, can take two possible choices, which will be in the mainframe of our analysis:

$\mathcal{M}_{1}=\operatorname{sgn}\left(f_{\mathrm{g}}\right)\left|f_{\mathrm{g}}\right|^{-1 / 2}$ and

$$
\mathcal{M}_{2}=\operatorname{sgn}\left(f_{\mathrm{p}}\right)\left|f_{\mathrm{p}}\right|^{-1 / 2} \text {. }
$$

These two differential equations are equivalent to Eq. (4), where $k_{\mathrm{r}}^{2}$ is an invariant ${ }^{4}$ of that family of equivalent differential equations. The determination of $\mathcal{M}_{i}$ presented previously, has been done under the consideration that $f_{\mathrm{g}}$ and $f_{\mathrm{p}}$ are real functions. In the case they are complex conjugates then $\mathcal{M}_{i}$ is defined as the real part of $f_{\mathrm{g}}$ and $f_{\mathrm{p}}$, respectively.

These complementary equations will be very useful in the analysis of the zeros of the wave function, $\Psi$. The location of the turning points will determine what is the most convenient formulation to study the properties of a given wave of frequency, $\omega$ and degree, $l$, i.e., the formulation is chosen such that $\mathcal{M}_{i}$ is strictly positive in the interval considered. In fact, the localization of the singular points depends on $\omega$ and $l$, and they correspond to the zeros of $f_{\mathrm{p}}=0$ or $f_{\mathrm{g}}=0$. Choosing the convenient self-adjoint formulation for a given wave of parameters $\omega$ and $l$, the function $\mathcal{P}_{i}^{-1}(r)$ is regular $\left(\mathcal{P}_{i}^{-1}(r) \neq \infty\right)$ in the interval considered. In this sense, in waves for which $\omega^{2}>\omega_{ \pm}^{2}\left(f_{\mathrm{p}}>0\right)$ in all the interval (acoustic type), the location of zeros of $\Psi$, corresponds to the location of zeros of $\mathcal{Y}_{1}$, provided that $\mathcal{M}_{1}$ does not have zeros on the interval considered. Equation (29) with $i=1$, is the convenient formulation to study the oscillatory motions. Conversely, in waves for which $\omega^{2}<\omega_{ \pm}^{2}\left(f_{\mathrm{g}}<0\right)$ in the interval considered (gravity type), the location of zeros of $\Psi$ corresponds to the location of zeros of $\mathcal{Y}_{2}$. Equation (29) with $i=2$, is then the convenient formulation to study the oscillatory motions. As we will discuss in Sect. 3.2, for some $l$ and $\omega$ a mixing mode can appear and both descriptions will need to be used. Under a correct choice of the self-adjoint form, the differential Eq. (4) for each of the propagative regions, the number and the location of the zeros of $\Psi$, can all be determined from a convenient self-adjoint form, even in such case.

In the following sections, when we are referring to a particular formulation, we will use the underscript $i$ on the variables $\mathcal{P}_{i}, \mathcal{Q}_{i}$ and $\mathcal{Y}_{i}$, with $i=1$ for the acoustic description and $i=2$ for the gravity description. This is a first step to the classification scheme that will be defined like it was originally proposed by Cowling (1941), taking into account the two driving mechanisms, the gravity and the pressure.

\subsection{Complementary phase equations}

The phase analysis proposed here, can be considered as a generalization of the method proposed by Eckart (1960), Scuflaire (1974), Osaki (1975), Gabriel \& Scuflaire (1979)

${ }^{4}$ In this context, invariant means that the different differential equations, that can be obtained by any type of transformation (of the independent and dependent variables or both), has $k_{\mathrm{r}}^{2}$ as a characteristic quantity, independent of the transformation considered. 

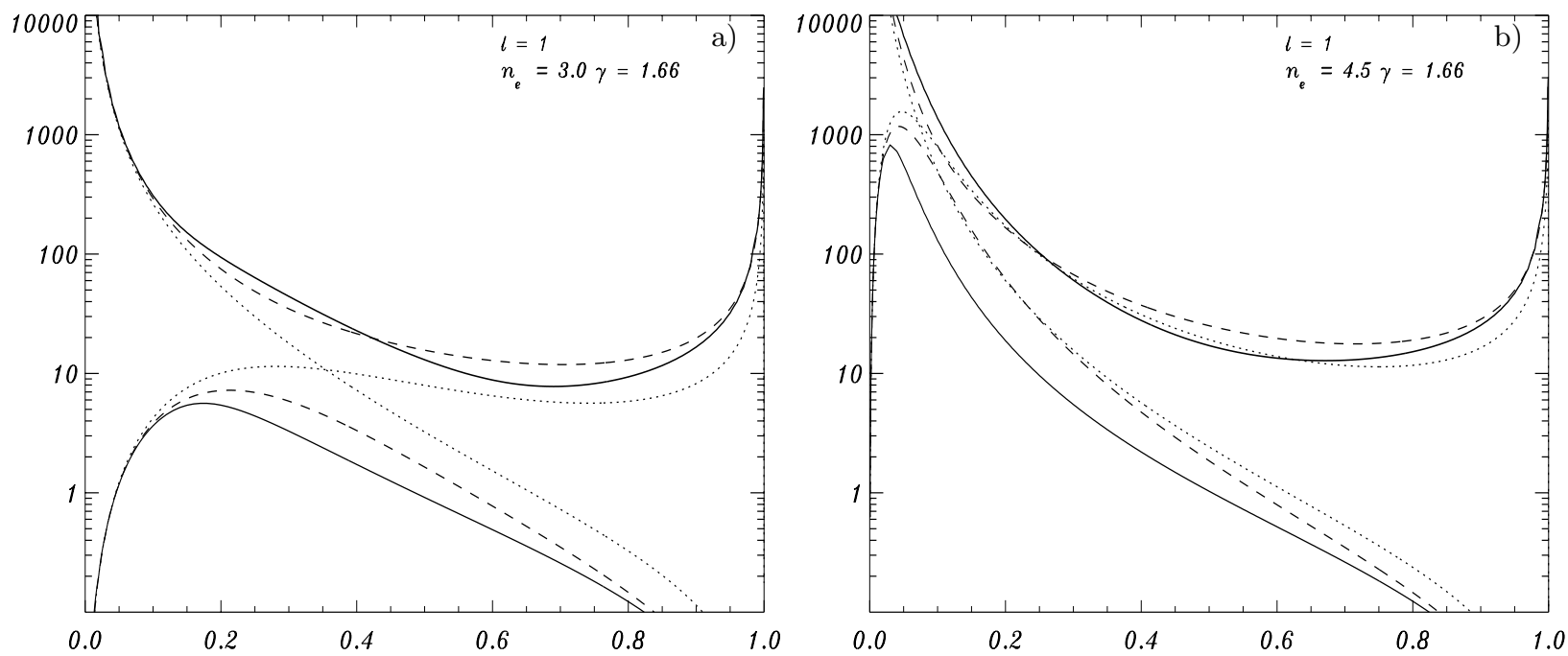

Fig. 2. Propagation diagram for Polytropic models of index $n_{\mathrm{e}}=3, n_{\mathrm{e}}=4.5$ and adiabatic index $\gamma=5 / 3$, for modes of degree $\ell=1$. The representation adopted is the same as in previous Fig. 1 .

and Gough (1993) to discuss the classification scheme of stellar oscillations. The method proposed by these authors is based on the algebraic counting of the number of nodes according to the behaviour of $p^{\prime}$ relatively to $\delta p$ (or the other two wave functions). In our case the classification scheme will be determined for a generic wave function that can be related with $\delta p$ (for example), and the order of the mode will be determined by the boundary conditions which can be, or not, reliable with the number of nodes of this wave function.

The motion equation for linear adiabatic nonradial oscillations (Eq. (4)) can be transformed into two selfadjoint differential equations (Eq. (29)) to determine the properties of the oscillations. Using the phase transformation (with $i=1,2$ ) for each of the self-adjoint forms (see details about this phase analysis in Appendix A), given by

$\mathcal{Y}_{i}=R_{i} \sin \theta_{i}$

and

$\mathcal{P}_{i} \mathcal{Y}_{i}^{\prime}=R_{i} \cos \theta_{i}$

where $R_{i}(\omega, l ; r)$ and $\theta_{i}(\omega, l ; r)$ are the amplitude and phase of the wave function $\mathcal{Y}_{i}$. After some straightforward manipulation, we obtain the following two first-order differential equations for the phase (phase equations):

$\frac{\mathrm{d} \theta_{1}}{\mathrm{~d} r}=\left(f_{\mathrm{p}}+\mathcal{T}\left(f_{\mathrm{g}}\right)\right) \sin ^{2} \theta_{1}+f_{\mathrm{g}} \cos ^{2} \theta_{1}$

and

$\frac{\mathrm{d} \theta_{2}}{\mathrm{~d} r}=\left(f_{\mathrm{g}}+\mathcal{T}\left(f_{\mathrm{p}}\right)\right) \sin ^{2} \theta_{2}+f_{\mathrm{p}} \cos ^{2} \theta_{2}$,

where $\mathcal{T}(x)$, is the real function given by

$\mathcal{T}(x)=\operatorname{sgn}(x)|x|^{-1 / 2} \frac{\mathrm{d}^{2}|x|^{-1 / 2}}{\mathrm{~d} r^{2}}$.

The amplitudes in each case, are given by

$R_{1}=R_{1, \mathrm{o}} \exp \left[\frac{1}{2} \int_{r_{\mathrm{o}}}^{r}\left(f_{\mathrm{g}}-f_{\mathrm{p}}-\mathcal{T}\left(f_{\mathrm{g}}\right)\right) \sin \left(2 \theta_{1}\right) \mathrm{d} r\right]$ and

$R_{2}=R_{2, \mathrm{o}} \exp \left[\frac{1}{2} \int_{r_{\mathrm{o}}}^{r}\left(f_{\mathrm{p}}-f_{\mathrm{g}}-\mathcal{T}\left(f_{\mathrm{p}}\right)\right) \sin \left(2 \theta_{2}\right) \mathrm{d} r\right]$

where $R_{i, \mathrm{o}}$ is the initial amplitude.

At this level, it is important to point out that Eqs. (36) and (37) are two different phase representations of the same physical system described by Eq. (4), written in a way that it avoids singular points for a given wave of degree $l$ and frequency $\omega$, in a given region. It is possible to determine a relation between the two phases, which is obtained by imposing a matching of the logarithmic derivatives of $\Psi$ (Eq. (28)) between both descriptions. This is given by

$\frac{\mathrm{d}}{\mathrm{d} r} \ln \left|\mathcal{M}_{1} \mathcal{Y}_{1}\right|=\frac{\mathrm{d}}{\mathrm{d} r} \ln \left|\mathcal{M}_{2} \mathcal{Y}_{2}\right|$

Using Eqs. (32) and (33) with the phase relation A.5, we obtain the following relation between phase $\theta_{1}$ and $\theta_{2}$ :

$\cot \theta_{1}=\frac{f_{\mathrm{p}}}{f_{\mathrm{g}}} \cot \theta_{2}-\frac{1}{2} \frac{1}{f_{\mathrm{g}}} \frac{\mathrm{d}}{\mathrm{d} r} \ln \left|\frac{f_{\mathrm{p}}}{f_{\mathrm{g}}}\right|$.

This relation can be used to determine the phase $\theta_{1}$ (of the acoustic description) knowing the phase $\theta_{2}$ (of the gravity description). We observe that when we want to convert $\theta_{2}$ into $\theta_{1}$ (go from the gravity description to the acoustic description), the point where $f_{\mathrm{g}}=0$ is singular, so that $\cot \theta_{1}=-\infty$, and consequently $\theta_{1}=0+n \pi$. Finally, we note that Eq. (42) is undefined in the radius where $f_{\mathrm{p}}=0$. However this situation never happens within this transformation.

In short, the propagation of a wave of degree $l$ and frequency $\omega$ can be explicitly determined for any of the three types of waves (which can be defined from the propagation diagrams) using the appropriate phase equation. For the pure acoustic type waves, the phase can be determined from Eq. (36) (acoustic description) because no singular points occur inside. 
For the pure gravity type waves, the phase can be determined by Eq. (37) (gravity description). Furthermore, for mixed type waves which correspond to a mode that propagates in two different regions, Eq. (36) can be used to determine the phase propagation on the gravity region and Eq. (37) to determine the phase propagation on the acoustic region.

The matching condition is made in the region where both descriptions, are accepted (without singular points). A possible choise is a point $r^{\star}$, where $f_{\mathrm{g}}\left(r^{\star}\right)=f_{\mathrm{p}}\left(r^{\star}\right)$ and $f_{\mathrm{g}}^{\prime}\left(r^{\star}\right)=f_{\mathrm{p}}^{\prime}\left(r^{\star}\right)$, in that case from Eq. (42) we obtain $\theta_{1}\left(r^{\star}\right)=\theta_{2}\left(r^{\star}\right)$. To make the matching we integrate each of the equations from each side of the interval and match them in the point $r=r^{\star}$, where both solutions are valid.

\subsection{Boundary conditions}

The stellar oscillation eigenmodes can be determined by using one of the phase equations (Eqs. (36) or (37)), with a convenient set of boundary conditions at the endpoints, the centre and the surface. The phase equations are equivalent one another and the choice of which discription to use is matter of the tast of the author as well the type of singularities that can be enconter. Indeed, a certain phase equation with the respective boundary conditions at the center and the surface, constitutes an eigen-value problem. In the following, we will indicate how it is possible to determine the boundary conditions for each representation.

The phase at the endpoints is determined by using one of two procedures. One consists in transforming the boundary conditions of the eigenfunction $\Psi$ or $\delta p$ (see Sect. 2.2) into an equivalent endpoint condition for the phase function $\theta_{i}$ of the phase associated system, related with the differential Eq. (29). Independent of the approximation made on the determination of the local wavenumber, defined on the equation of motion (see Sect. 2), it is possible to determine the phase at the endpoints from the Lagrangian perturbation of pressure $\delta p$ and the Eq. (A.5). In the Appendix B, we present the determination of the boundary condition in the case of the planar approximation (Deubner \& Gough 1984).

An alternatively method consists in determining the phase at the endpoints by imposing the regularity of the phase function $\theta_{i}$. A detailed analysis of the phase Eqs. (36) and (37), indicates that these ones are singular at the endpoints, $r=0$ (for all stars) and $r=R$ (only for polytropes). This implies that the boundary conditions must be applied such that the singular solution at each of the endpoints is eliminated. This is obtained by expanding the solution, $\theta_{i}$, as a power series of $r$, relatively to the critical endpoint. It is necessary also to expand the equilibrium quantities around the endpoints, $r=0$ and $r=R$. It is worth to notice that for each description it is possible to study the properties of the gravity waves, as well as of the acoustic waves, even if it is more convenient to use a gravity description to study the eigenstates of gravity perturbations and similarly the acoustic description to study the eigenstates of acoustic perturbations. In that case for each description, we will refered to the perturbation as characterized by the main contributer for the restoring force that is present in a certain region inside the star. In this context, we will refered to an acoustic behaviour or the gravity behaviour, as it is the gravity or the pressure the main contributer for the restoring force. The mixed modes can be treated as a combination of these two "asymptotic" cases (as discussed in the previous section), as an acoustic type mode or gravity type mode for each of the region where one of these character prevails. In particular, the inner and outer boundary conditions for mixed modes are determined based in which is the dominant wave character near the center or near the surface of the star.

The inner boundary condition near the center is obtained by imposing that the solution to the phase equation is regular, i.e. $\theta_{i}$ has a finite value. Now, we will illustrate that by considering the case corresponding to the acoustic description, i.e., we are interested in determine the solution, $\theta_{1}$ as given by Eq. (36). Furthermore, we will starting by considering a wave that has an acoustic behaviour near the stellar center, it follows that $\theta_{1}$ must be a regular function at the center, as the phase has a finite value. We notice that the first-member of Eq. (36) is singular, consequently, the finite value of $\mathrm{d} \theta_{1} / \mathrm{d} r$ is obtained by choosing all the multi-value solutions, such as $\theta_{1}=0+n \pi$ where $n$ is a positive or a negative integer (or in an equivalent form $\theta_{1}=0(\bmod \pi)$; see also Appendix B). Similary in the case of the gravity description (Eq. (36)), a wave with a gravity behaviour at the center has a regular solution if $\theta_{2}=0(\bmod \pi)$. It follows that the same boundary conditions can be presented in both descriptions, given that $\theta_{1}=\theta_{2}+\pi / 2$. This relation between phases is illustrated in the next section. In resume, the boundary condition for the center require that

$$
\left(\frac{\mathrm{d} \theta_{i}}{\mathrm{~d} r}\right)_{r=0}=\left\{\begin{array}{l}
0(\bmod \pi) \\
+\infty(\bmod \pi)
\end{array} .\right.
$$

Alternativelly, from the phase Eqs. (36) and (37) it follows in the case of the acoustic description,

$$
\theta_{1}(\omega, l ; 0)=\left\{\begin{array}{l}
0(\bmod \pi) \text { acoustic behaviour } \\
\frac{\pi}{2}(\bmod \pi) \text { gravity behaviour }
\end{array}\right.
$$

for modes with acoustic and gravity behaviour near the centre of the star, and in the case of the gravity description,

$\theta_{2}(\omega, l ; 0)= \begin{cases}\frac{\pi}{2}(\bmod \pi) & \text { acoustic behaviour } \\ 0(\bmod \pi) & \text { gravity behaviour }\end{cases}$

for modes with acoustic or gravity behaviour.

The outer boundary condition, can be obtained also by a series expansion around the endpoint $r=R$. In the case 
of polytropic equilibrium structures, because the sound speed is zero at $r=R$, the phase Eqs. (36) and (37) can be singular. It follows that

$$
\left(\frac{\mathrm{d} \theta_{i}}{\mathrm{~d} r}\right)_{r=R}=\left\{\begin{array}{c}
0(\bmod \pi) \\
+\infty(\bmod \pi)
\end{array},\right.
$$

then we obtain the following type of conditions for the surface,

$\tan ^{2} \theta_{1}(\omega, l ; R)=-\frac{f_{\mathrm{g}}}{f_{\mathrm{p}}+\mathcal{T}\left(f_{\mathrm{g}}\right)}(\bmod \pi)$

and

$\tan ^{2} \theta_{2}(\omega, l ; R)=-\frac{f_{\mathrm{p}}}{f_{\mathrm{g}}+\mathcal{T}\left(f_{\mathrm{p}}\right)}(\bmod \pi)$.

An approximative solution can be obtain by using the results of Appendix B. We start bu notice, that the sound speed vanish at the surface, consequently, we have $\tan ^{2} \theta_{1} \sim-\left(1-N^{2} \omega^{-2}\right)$ and $\tan ^{2} \theta_{2} \sim-1 /\left(1-N^{2} \omega^{-2}\right)$. In case of acoustic description if we consider the asymptotic limit, $\omega \rightarrow \infty$, then $\tan ^{2} \theta_{1}=1$, or $\theta_{1}=0(\bmod \pi)$ and in the case of gravity description if we consider the asymptotic limit $\omega \rightarrow 0$ then $\tan ^{2} \theta_{2}=1$ or $\theta_{2}=0(\bmod \pi)$. Consequently, following a similar procedure to study the solution in the center of the star, we can obtain the expression for the two type of waves in both descriptions. It follows,

$\theta_{1}(\omega, l ; R) \rightarrow\left\{\begin{array}{l}0(\bmod \pi) \text { acoustic behaviour } \\ \frac{\pi}{2}(\bmod \pi) \text { gravity behaviour }\end{array}\right.$

and

$$
\theta_{2}(\omega, l ; R) \rightarrow\left\{\begin{array}{l}
\frac{\pi}{2}(\bmod \pi) \text { acoustic behaviour } \\
0(\bmod \pi) \text { gravity behaviour }
\end{array}\right.
$$

In real stars this outer boundary condition can be slightly different, but the main conclusion remains the same.

We observe that with these boundary conditions for the inner endpoint, in both descriptions both solutions are regular because the amplitude and the phase have finite values for $r=0$.

\subsection{Simplified phase equations}

Ledoux \& Walraven (1958) have derived a particular solution, which presents two asymptotic limits for very high and very small eigenfrequencies. In this case the system of stellar oscillations under the Cowling approximation tends toward a Sturm-Liouville type solution. Here, an analogous situation takes place, when the term with the form $\mathcal{T}(x)$, in Eqs. (36) and (37) can be neglected. This is a good approximation in most of the stellar interior, because this term remains small compared with the other terms almost everywhere, except near the turning points (where $f_{\mathrm{p}}$ or $f_{\mathrm{g}}$ becomes 0 ) and the stellar surface. In that case, the nodes of $\mathcal{Y}_{1}$ (or $\mathcal{Y}_{2}$ ) correspond to extremes of $\mathcal{Y}_{2}$ (or $\left.\mathcal{Y}_{1}\right)$. The phase equations take the simple form:

$\frac{\mathrm{d} \theta_{1}}{\mathrm{~d} r}=f_{\mathrm{p}} \sin ^{2} \theta_{1}+f_{\mathrm{g}} \cos ^{2} \theta_{1}$

and

$\frac{\mathrm{d} \theta_{2}}{\mathrm{~d} r}=f_{\mathrm{g}} \sin ^{2} \theta_{2}+f_{\mathrm{p}} \cos ^{2} \theta_{2}$.

In that case the phase between the two prescriptions has the very simple relation, $\theta_{1}=\theta_{2} \pm \pi / 2$. It is also important to remark that, in a given representation when a solution at the initial endpoint is singular, it is regular at the other phase representation. This lead us to use a convenient phase repesentation to avoid this singular behaviour, which is the origin of the acoustic and gravity description presented in the last section.

It is important to stress that this is a very good representation for the phase equations and from that we can say that the difference between the acoustic and gravity waves presents a shift of $\pi / 2$. In this case, it is very clear the fact that both phase equations are strictly equivalent.

For a given $l$, if $\omega^{2}$ is large this suggests the existence of a spectrum of indefinitely increasing eigenvalues, corresponding to the eigensolution denoted by acoustic modes or $p$-modes. Conversely, for $\omega^{2}$ very small, this tends to define a Sturm-Liouville problem with the parameter $1 / \omega^{2}$, which corresponds to the eigensolutions denoted by gravity modes or $g$-modes.

\section{Propagation of acoustic-gravity waves}

The stellar oscillations are now described by one or another phase equations (Eqs. (36) and (37)). These phase equations with the respective boundary conditions 44 and 47 or 45 and 48 form a boundary value problem with $\omega^{2}$ as an eigenvalue. In this section we start by determining numerically the eigenfrequencies that are solutions of the eigenvalue problem presented. By the following, we will present a classification scheme base in the result obtained.

Let's start by observing that the phase function $\theta_{i}(\omega, l, r)(\bmod \pi)$, has the same number of zeros at the same location as the wave function $\Psi$, provided that the transformations are made as was indicated previously. However, the number and location of the zeros of the wave function, $\delta p$, (obtained from the equation of motion, Sect. 2), are the same as $\Psi$, only for pure acoustic or gravity modes, due to the fact that the discriminant $f$ as given by Eq. (10) is strictly positive or negative in all the star. In the case of mixed modes, another zero occurs for the wave function, $\delta p$, at the radius of the star where $f=0$.

The main properties of the stellar oscillations, can be well defined in a linear phase diagram (see Fig. 4) that determines the phase dependence of a given eigenmode with the radius, for a given equilibrium structure. The construction of the linear phase diagram is made by introduction of an effective phase, $\phi(\omega, l ; r)$. This effective 


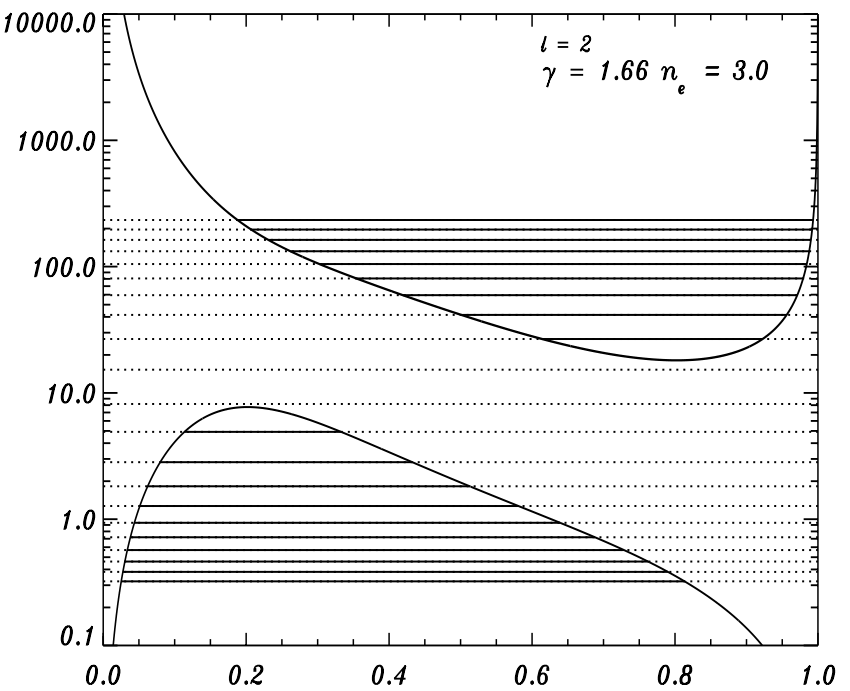

Fig. 3. Propagation diagram for a polytropic model with index $n_{\mathrm{e}}=3.0$, adiabatic index $\gamma=5 / 3$ and modes of degree $l=2$. The solid curves represent the critical frequencies $\omega_{ \pm}$, delimiting the two propagation regions of the stellar oscillations as it is obtained from the first Cowling approximation motion equation (see text). The eigenstates corresponding to the eigenmodes with dimensionless square of the circular frequency, $\omega^{2}: 234,196,163,132.4,104.8,80.55,59.42,41.47$, $26.72,15.26,8.175,4.915,2.828,1.822,1.270,0.9360,0.7188$.

phase for each eigenmode, of an eigenvalue $\omega$ and degree $l$, is given by

$\phi(\omega, l ; r)=\theta_{i}(\omega, l ; r)-\theta_{i}(\omega, l ; 0)$

where $\theta_{i}$, is the phase function. The computation of the linear phase diagram is made using the phase Eq. (36) in the case of acoustic modes, and the Eq. (37), with $\theta_{2}=\theta_{1} \pm \pi / 2$, in the case of gravity modes. In that case the linear phase diagram for acoustic and gravity modes is constructed based in the phase $\theta_{1}$. This is justified by the simple relation between acoustic and gravity description when the function $\mathcal{T}$ is neglected in the phase equation. We remember that an eigenmode is the product of an amplitude function by a phase function, and the main properties of the eigenmode can be discussed using the phase equation.

The nonradial oscillations of a star can be interpreted as a superposition of stationary waves generated by the competition between the pressure and the gravity. These two driving mechanisms, define the two propagation regions illustrated in the Fig. 3 for a polytropic equilibrium structure of index $n_{\mathrm{e}}=3$. An eigenstate, can be interpreted heuristically as being the result of the competition between these two propagative regions, as it is determined by the phase Eq. (36) (or Eq. (37)). The eigenstates corresponding to the acoustic waves of high frequency, can be determined neglecting the contribution of the gravity propagation region (which corresponds to neglecting the second term of Eq. (36), which case is similar to the Sturm-Liouville problem. In this case, the modes form a well-ordered sequence, starting from a given fundamental state. Conversely, the eigenstates of gravity waves of very low frequency, can be determined by neglecting the contribution of the acoustic propagation. A particular state occurs in the star, even in a very simple equilibrium structure, caused by the fact that both propagative regions contribute in the same order of magnitude for this standing wave. This corresponds to the $f$-mode. Furthermore, this eigenmode presents a mixed nature and propagates mainly or uniquely in the evanescent region of the star. Cowling (1941) was the first to point out the fundamental difference of nature of the $f$-mode, relatively to the $p$-modes and $g$-modes. The $f$-mode corresponds to the fundamental oscillation of the system for which no nodes occur on the wavefunction. Furthermore, for stars where mixing of modes can occur, this $f$-mode, as it is defined here, cannot exist.

It is possible to prove that the modes form a wellordered sequence when the star is simple ${ }^{5}$, given that two different eigenmodes never cross. However, this can be guaranteed only for modes that propagate locally as an acoustic type wave or gravity type wave. The evanescent type mode $f$ is the only exception. The fact that the phase of two eigenmodes never crosses, is reliable on the topological properties of the propagation diagram (see Figs. 1, 2 and 3) and the properties of the phase equations. For two waves with frequencies, $\omega_{a}$ and $\omega_{b}$, such as $\omega_{b}<\omega_{a}$, the propagative characteristics are fixed differently for an acoustic-gravity wave, in function of the type of propagation. For a wave that propagates acoustically, the higher frequency presents always a larger propagative region. Conversely, for a wave that propagates by gravity type, the higher frequency presents always a smaller propagative region. Furthermore, on the propagative region, we have that $f_{\mathrm{p}}\left(r ; \omega_{a}\right) \geq f_{\mathrm{p}}\left(r ; \omega_{b}\right) \geq f_{\mathrm{g}}\left(r ; \omega_{a}\right) \geq f_{\mathrm{g}}\left(r ; \omega_{b}\right) \geq 0$, for two waves that propagate acoustically, and $f_{\mathrm{g}}\left(r ; \omega_{a}\right) \leq$ $f_{\mathrm{g}}\left(r ; \omega_{b}\right) \leq f_{\mathrm{p}}\left(r ; \omega_{a}\right) \leq f_{\mathrm{p}}\left(r ; \omega_{b}\right) \leq 0$, on the other cases. Using the phase equations corresponding to each one of these descriptions, we have that $\theta_{1, a}(r) \geq \theta_{1, b}(r)$ for acoustic type propagation and $\theta_{2, a}(r) \leq \theta_{2, b}(r)$ for gravity type propagation, for each point inside the star. This determines that two eigenmodes can never cross each other, if they are acoustic waves, gravity waves or mixed waves.

In Figs. 3 and 4 we represent the propagation diagram and the linear phase diagram for the eigenmodes of degree $l$, for a polytropic equilibrium structure of index $n_{\mathrm{e}}=3$ and adiabatic index $\gamma=5 / 3$. The phase function of acoustic waves and gravity waves, present important propagative topological differences between them. However, for the very low frequency acoustic waves and high frequency gravity waves, the propagation is similar to a $f$-mode.

The main asymptotic properties can be illustrated in the planar approximation (see Appendix B). In the case of acoustic modes, corresponding to the higher frequencies $(\omega \rightarrow+\infty)$, the phase Eq. (36) is dominated by the term

\footnotetext{
5 A star is considered simple in this context: when its propagation diagram can be obtained by a topological deformation of a simple polytrope such as polytrope $n_{\mathrm{e}}=3$.
} 


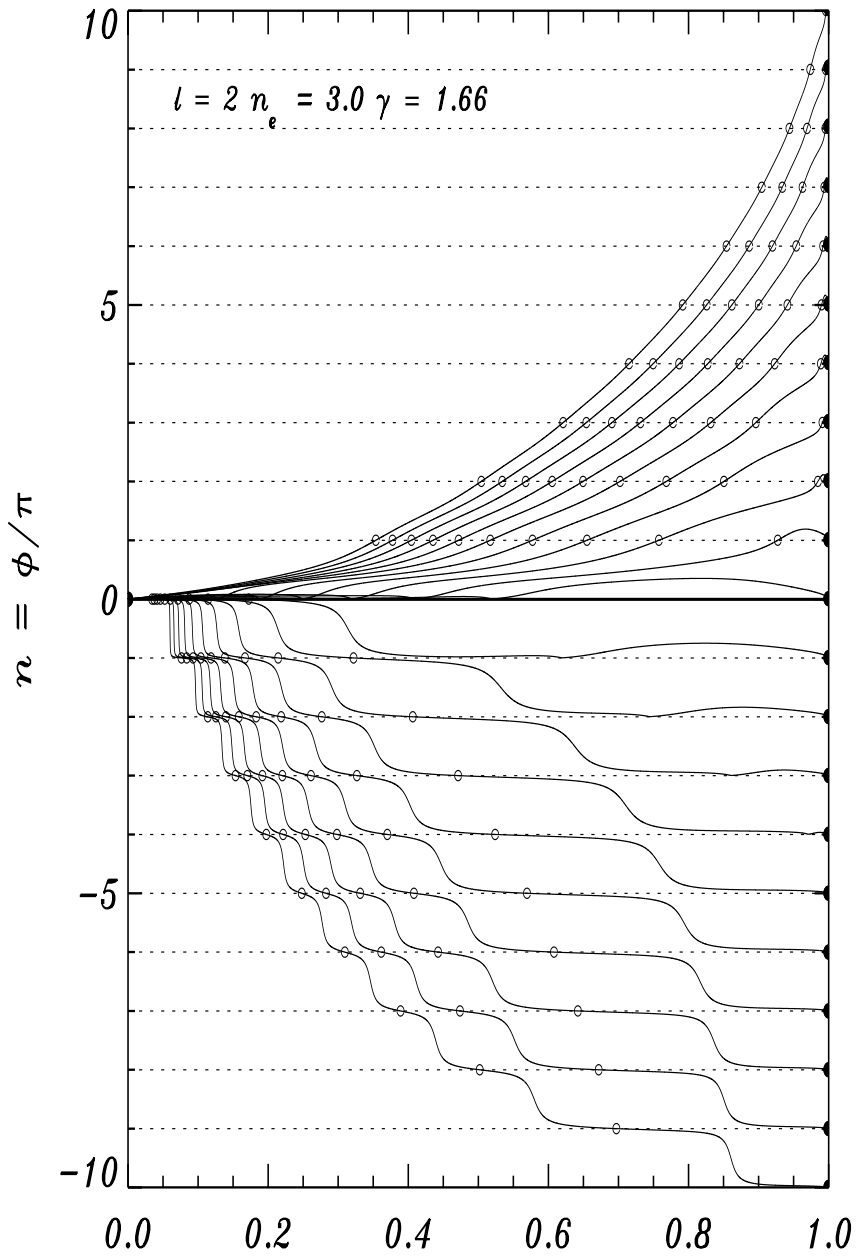

Fig. 4. Linear phase diagram for a polytropic equilibrium model of index, $n_{\mathrm{e}}=3$, and adiabatic index $\gamma=5 / 3$. The curves represent the phase function $\phi(\omega, l, r)$ of eigenmodes of frequency $\omega$ and degree $l=2$. The thin-dashed straight lines represent the number $\pi$-cycle that a phase function has developed from the internal endpoint $r=0$, until the external endpoint $r=R$. The inner boundary condition at $r=0$ and the outer boundary condition at $r=R$ for the different cycles of $\pi$ are represented by a black dot. In the propagative regions where the eigenmodes behave like an acoustic wave the phase increases (clockwise direction) and in propagative regions where the eigenmodes behave like a gravity wave the phase decreases (anticlockwise direction). The phase function, $\phi$ is calculated for a given eigenstate, from the higher to the lower eigenfrequencies. The radial order number, $n=\phi / \pi$, takes values from 10 to -10 (see text).

$\omega / c$, through the discriminant $f_{\mathrm{p}}$ and $f_{\mathrm{g}}$. Then the phase, $\theta_{1}$, is given approximatively by

$\theta_{1}(\omega, l ; r) \approx \omega \tau \quad \tau(r)=\int_{0}^{r} \frac{\mathrm{d} r}{c}$

where $\tau$, is the so-called acoustic radius. However, the phase stays practically constant on the evanescent regions.

Alternatively, the gravity modes are given by Eq. (37), corresponding to the smaller frequencies $(\omega \rightarrow 0)$, the discriminants $f_{\mathrm{p}}$ and $f_{\mathrm{g}}$ can be approximated by

$f_{\mathrm{p}} \approx-\frac{L^{2}}{\omega} \frac{c}{r^{2}}$ and $f_{\mathrm{g}} \approx-\frac{1}{\omega} \frac{N^{2}}{c}$.

In that case, we have a competition between these two terms, which give the waviness pattern, characteristic of the phase function of gravity waves. It is possible to demonstrate that the asymptotic leading term, in this case leads to $\sqrt{f_{\mathrm{p}} f_{\mathrm{g}}} \approx L / \omega N / r$.

In the example presented above, mixing of modes does not occur (no overlap of the propagative region), but if this happens, the phase function presents a similar behaviour to the acoustic and gravity modes for each one of the respective propagation regions (see Fig. $2 \mathrm{~b}$ ).

\section{Classification of stellar oscillations}

To discuss the differences between the new classification scheme proposed in this work relatively to the classification schemes normally used, we present in the following the original classification proposed by Cowling (1941) and the generalization of that one made by Eckart (1960), Scuflaire (1974) and Osaki (1975).

\subsection{Classical classification schemes}

The first classification scheme to label the eigenmodes of oscillations was introduced by T. Cowling (1941). This is valid provided that the reduction of the fourth-order system of nonradial adiabatic stellar oscillations to a secondorder system is obtained under the hypothesis that the Eulerian perturbation of the gravitational field can be ignored. This scheme provides a good qualitative description of higher-order modes for $l=0$ and $l=1$ and for all the modes with $l \geq 2$.

Formally, the frequency is unbounded below (except when $l=0$ ), and also unbounded above. Therefore, it is not immediately obvious where to choose the origin of $n$. It is possible to choose $n$ such that as $n \rightarrow \pm \infty$ for a fixed $l,|n|-1$ is the number of zeros in the eigenfunction, when $l \geq 2$ and $|n|$ is the number of zeros in the eigenfunction, when $l=0$. For spherically symmetrical modes $(l=0)$, the lowest-frequency mode (fundamental mode) is labeled $n=1$. In simple stellar models, modes with $n>0$ and $n<0$ have the characteristics of acoustic and internal gravity waves; they were designated $p$-modes and $g$-modes by Cowling (1940). Modes with $n=0$, are fundamental $g$-modes or $f$-mode, and have the property that when $l \gg 1$, they have no zeros in the eigenfunction. Under these considerations, the modes of a star can be classified as $p$-modes for $n>0$ and $g$-modes for $n<0$, and a (unique) $f$-mode for $n=0$. Then both $g$ and $p$ sequences start from $n=1$. The fundamental $l=0$ mode, was labeled with $n=1$.

The Cowling classification scheme has been generalized by Eckart (1960), by taking into account the tunnel effect that can take place when the competition between the two restoring forces pressure and gravity is of the same 
order. Eckart (1960) discusses how the phase differences between vertical displacement and pressure fluctuation decreases with height for acoustic modes and increase for $g$-modes, so that order $n$ can be computed by first assigning a signal to each zero in the eigenfunction according to the direction of variation of the phase difference, and then counting the zeros algebraically. Scuflaire (1974) and Osaki (1975) presented the same criterion to classify stellar oscillations. Scuflaire (1974) has demonstrated that, for relatively condensed polytropic modes $n_{\mathrm{e}} \geq 3.4213$ (in case of modes of degree $l=2$ ) and $\gamma=5 / 3$, the mixing of modes (in the Cowling approximation) occurs. This classification scheme, has been found to work well in most stars for oscillations with $l \geq 2$, when the Cowling approximation is not considered. The problem remains for $l=1$. Another classification scheme was proposed by Shibahashi \& Osaki (1976), based on the properties of the wave on the main trapping region.

However, none of these classification schemes is sufficiently general. This is due to two main reasons. One, is the fact that the classification scheme is dependent on the eigenfunction used to determine the order $n$ of the mode, which in the most general case does not necessarily have the same number of zeros of other eigenfunctions choosen also to classify the modes of some physical system (Cox 1980). This means that the classification scheme depends on the properties of the wave function choosen to define the system, as it has been illustrated by different authors (Cox 1980 and references there). The classification scheme does not work for modes with $l \leq 1$. Even if we consider that this hypothesis is acceptable, there is a second problem which remains. It is the fact that all the classification scheme has been done under the Cowling approximation, and the full problem is just an extension of this case.

\subsection{New classification scheme}

The resonant cavities are clearly defined for all modes of any order, and not only for modes of high-order and very high degree, as is normally done in the most classical scheme classifications. We start by pointing out, that the phase functions $\theta_{i}(l, \omega ; r)$, only depend on the structure of the equation of motion, i.e. on the topology of the propagation diagram.

The oscillation corresponding to an eigenstate, can be defined in terms of the boundary conditions, where $\omega^{2}$ is a parameter. In this sense, an eigenstate occurs when the difference of phase between the initial phase at $r=0$ and the final phase ${ }^{6}$ at $r=R$, are a multiple of $\pi$. This introduces a natural scheme classification for the eigenmodes, which determines the order number of the mode, $n$, as the integer associated to the total number of $\pi$-cycles that

\footnotetext{
${ }^{6}$ For some modes the influence of the boundary conditions is negligible. In those cases only the layers of the evanescent region, very near the turning point, contribute to the phase. Such cases, correspond to consider that the endpoints of the problem are: $-\infty$ and $+\infty$.
}

the phase function has developed from the inner endpoint where the inner boundary condition is fixed until the outer endpoint where the outer boundary condition is fixed (see Sect. 4). The determination of the eigenvalue equation, similar to the Bohr-Sommerfeld quantization rule, is done in one particular description given that, as we mentioned previously, both descriptions are equivalent. An eigenstate corresponds to the following condition for each of the wave types:

$\phi(\omega, l, R)=\theta_{i}(\omega, l ; R)-\theta_{i}(\omega, l ; 0)=n \pi$

where $\phi(\omega ; R)$ is the difference between the outer boundary condition fixed for $r=R$, given by $\theta_{i}(\omega, l ; R)$ and the inner boundary condition given by $\theta_{i}(\omega, l ; 0)$. In our case it is given by the expression 47 (or 48 ). $n$, is a negative or positive integer, starting from 0 . The well-ordered sequence $^{7}$ of eigenfrequencies, corresponding to the ordering of the integer $n$, from $-\infty$ to $+\infty$, is guaranteed by the properties of the phase function discussed in the last section. The only exception is the unique $f$-mode. It will be positive, if $\theta_{i}(R, \omega)>\theta_{i}(0, \omega)$ and it will be negative in the opposite case. The negative integer is reserved for the gravity modes, and the positive integer for the acoustic modes. However in the case of mixed modes the sign of $n$, determines the dominant character of the propagation mode. This classification scheme is general and is fixed by the boundary conditions, and does not depend on the turning points of the modes, or even on the algebraic counting of the number of nodes of the eigenfunction (i.e. the number of $\pi$ cycles, that the phase function has developed from the internal endpoint, until the external endpoint).

The more condensed polytropes (see Fig. 2b) cannot have the $f$-mode. However, the degeneracy of the eigenstates of low order modes can occur more frequently due to the overlap of the two propagative regions. In Figs. 3 and 4 we present the propagation diagram and the linear phase diagram of a polytope of index $n_{\mathrm{e}}=3$. The high values of $|n|$ correspond to pure acoustic waves for positive values of $n$ and pure gravity waves for negative values of $n$. This occurs because these waves present only one propagative region and a well behaved phase function in each case. In that case, the main properties are conserved for modes of low radial order. However, in the case of condensed polytropes such as polytropes of index $n_{\mathrm{e}}=4.5$, for the lower radial order modes, the wave function presents a mixed character which is determined by each of the two propagative regions. In this case the eigenstate can occur by two possible ways: or the eigenstate is mainly determined in only one of the propagative regions, or is fixed by both propagative regions when the evanescent region between them is small compared with the wavelength of the wave, i.e., tunnel effect. It will be the particular topological nature of the propagation diagram that will determine the different eigenstates. In such cases the previous

\footnotetext{
7 by "well-ordered sequence" we mean that two modes of different frequencies never cross.
} 


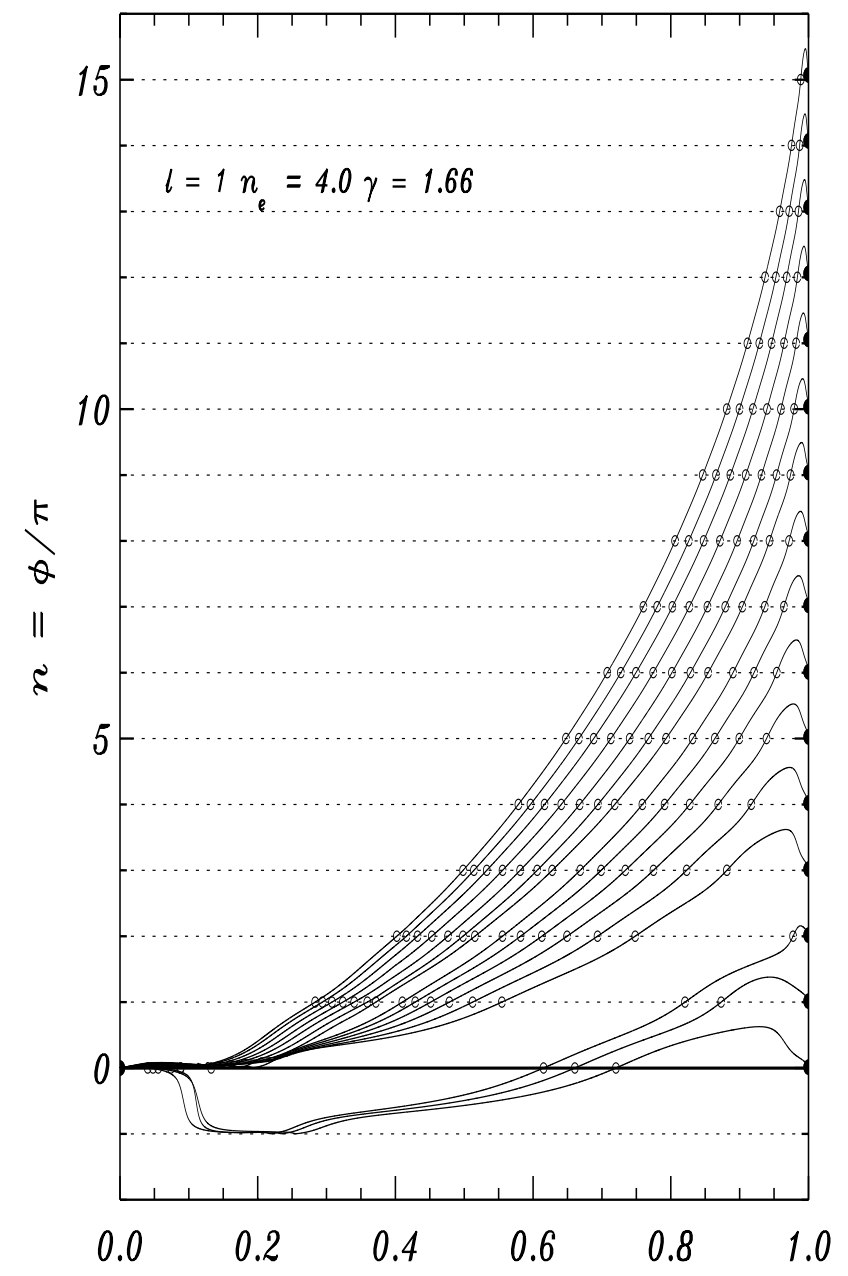

Fig. 5. Linear phase diagram of modes of degree $l=1$, for a polytropic model of index, $n_{\mathrm{e}}=4$ and adiabatic index $\gamma=5 / 3$. The representation adopted is the same of the previous figure.

classification fails for modes of low degree, particularly those with $l=1$, in highly condensed stellar models. In the scheme presented, the dipole modes have a perfect ordering, even for the lowest orders, such as $n \leq 2$. This is evident for modes of a polytrope of index $n_{\mathrm{e}}=4$ with $n \leq 3, l=1$ (see Fig. 5). The lower-order modes have phase paths quite different from those of the higher-order modes, because the perturbation to the gravitational potential in the central region can change the character, in regions where the mode behaves like a gravity wave. We believe that this is the reason why previous classification schemes do not work. This behaviour is likely to occur in real stars (Lopes 2000).

It is convenient, also to point out the agreement between this method and the classical method proposed by Cowling (1941) and others, for the higher order modes of simple stars (see Fig. 4). For example, for a polytrope of $n_{\mathrm{e}}=3$ the eigenstate of order $n=10$, corresponds to an acoustic mode, for which the phase function, presents 11 nodes (i.e. $\pi$-cycles of the phase function) and is classified with $n=10$ in the classical scheme. A gravity wave, of order $n=6$ presents 7 nodes and is classified with $n=6$ in the classical scheme.

Finally, we observe that only in the case of a SturmLiouville eigenvalue type problem, it is guaranteed that the eigenfunction associated to some eigenvalues, has exactly $n$ zeros (Cowling 1941). It is just in eigenvalue problems similar to that one that it is possible to use the counting of the zeros of the eigenfunction to label the order of the eigenstate. Moreover, the algebraic counting of zeros of the eigenfunction on the gravity region or the acoustic region can also be used to label the states of the system (Scuflaire 1974). This is on the basis of the usual classification schemes, and it is one of the reasons why they do not work.

\section{Summary and conclusion}

It has been known since the first classification scheme proposed by Cowling (1941) and later developed by Scuflaire (1974) and Osaki (1975), that the classification of modes for relatively evolved stars is ambiguous. This problem in the classification arises from the fact that the complete fourth-order system of stellar nonradial adiabatic oscillations has been obtained by a simple extension of the classification scheme proposed by Cowling (1941) for a second-order system, obtained by neglecting the Eulerian perturbation of the gravitational potential in the full problem. Furthermore, any classification scheme depends on the wave function choosen to determine the eigenstates of the oscillatory system and on its number of zeros.

In this work, we have addressed the problem determining a unique classification scheme for the equation of motion, independent of the eigenfunction used to characterize the oscillatory motion and its number of zeros.

The principle consists in determining two self-adjoint forms of the equation of motion, build by using the propagation diagram which is associated with the different types of stellar perturbations, that overcome from the restoring force due to the competition between the pressure and the gravity. All the properties of the eigenmodes depend only on the topological properties of the propagation diagram. For each of the self-adjoint forms of the equation of motion, a phase representation of the eigenmode is made which consists in describing the oscillatory motion of adiabatic oscillations by a system of two first-order nonlinear differential equations for the phase and the amplitude. The equations are coupled only in the amplitude equation, leaving a single phase equation to determine the eigenfrequencies. A convenient phase representation is determined for each type of propagation, corresponding to each case, whether is the pressure or the gravity that dominates in the restoring force. This competition between the pressure and gravity, can generate 3 types of waves for which the local properties can be defined on a propagation diagram together with the respective linear phase diagram, for which the linear phase properties are distinct from each other. Furthermore, the linear phase diagram proposed, constitutes a powerful method to determine an unique radial 
order number $n$. Similarly to the classical methods, the sign of order number $n$ determines the dominant nature of the wave.

A possible atempt to classified the eigenvalues of the linear adiabatic nonradial oscillation system (of the fourth-order problem of nonradial adiabatic oscillations), is to determine the radial order $n$ of the eigenmode by using the phase equations Eqs. (36) and (37) with the respective boundary conditions given by Eqs. (44) and (47) or (45) and (48). In that way, this technique can be used to constrain the linear phase diagram, which can determine the unique radial order number $n$. However, it is expected that this technique will not work in all the cases, once the second-order system have been obtained by an approximative method. A more extensive study must be made to identify those cases that this porcedure can be applied.

Finally, the classical scheme proposed by Scuflaire (1974) and Osaki (1975) corresponds to a particular case of this general scheme, for which the order number $n$ is equal to the algebraic counting of the number of modes in the different propagative regions, with a positive sign for modes in the acoustic propagative region and a negative sign for modes in the gravity propagative region.

Acknowledgements. Ilídio Lopes thanks Douglas Gough for stimulating discussions on the oscillatory properties of acoustic waves and stellar modelling. He also acknowledges support of a grant from the Particle Physics and Astronomy Research Council (UK). Ilídio Lopes would like to thank the referee J. Provost for the careful reading of the paper as well the valuable comments that have allow me to improve the original manuscript.

\section{Appendix A: Phase analysis}

Here, we introduce a technique which allows to investigate the properties of the eigen-solutions of a formally self-adjoint differential equation, which was been developed initially, to analyze the properties of the solutions of the Sturm-Liouville problem (Prüfer 1926). This method is convenient to find out how often the solution of Eq. (29) (corresponding to both descriptions), oscillates in the interval under consideration, which corresponds to the number of zeros of that solution.

\section{A.1. Basic transformation}

The linear adiabatic wave equation of stellar oscillations (Eq. (29)), is written as a system of two differential equations of first-order. This is accomplished by the transformation

$\mathcal{Z}_{i}(r)=\frac{1}{\mathcal{P}_{i}(r)} \frac{\mathrm{d} \mathcal{Y}_{i}}{\mathrm{~d} r}$

resulting in the system

$$
\left\{\begin{aligned}
\frac{\mathrm{d} \mathcal{Y}_{i}}{\mathrm{~d} r} & =\mathcal{P}_{i}^{-1}(r) \mathcal{Z}_{i} \\
\frac{\mathrm{d} \mathcal{Z}_{i}}{\mathrm{~d} r} & =-\mathcal{Q}_{i}(r) \mathcal{Y}_{i}
\end{aligned}\right.
$$

It is possible to reduce this system to a new one, where the two equations are coupled only in the amplitude equation, leaving a single phase equation to determine the eigenfrequencies. This is accomplished by using polar coordinates (phase transformation),

$\mathcal{Y}_{i}=R_{i} \sin \theta_{i} \quad \mathcal{Z}_{i}=\mathcal{P}_{i} \mathcal{Y}_{i}^{\prime}=R_{i} \cos \theta_{i}$

where $\mathcal{R}_{i}=R_{i}(r)$, is the amplitude of the wave function $\mathcal{Y}_{i}$, given by

$R_{i}^{2}=\mathcal{Y}_{i}^{2}+\left(\mathcal{P}_{i} \mathcal{Y}_{i}^{\prime}\right)^{2}$

and $\theta_{i}=\theta_{i}(r)$, the phase of the wave function $\mathcal{Y}_{i}$, given by

$\theta_{i}=\arctan \left(\frac{\mathcal{Y}_{i}}{\mathcal{P}_{i} \mathcal{Y}_{i}^{\prime}}\right)$

Solving the system (A.2) for $R_{i}$ and $\theta_{i}$, yields

$\frac{\mathrm{d} \theta_{i}}{\mathrm{~d} r}=\mathcal{Q}_{i}(r) \sin ^{2} \theta_{i}+\frac{1}{\mathcal{P}_{i}(r)} \cos ^{2} \theta_{i}$

and

$R_{i}=R_{i, \mathrm{o}} \exp \left[\frac{1}{2} \int_{r_{\mathrm{o}}}^{r}\left(\frac{1}{\mathcal{P}_{i}(r)}-\mathcal{Q}_{i}(r)\right) \sin \left(2 \theta_{i}\right) \mathrm{d} r\right]$.

Equations (A.6) and (A.7) are called the phase associated system. Each solution of the original self-adjoint differential Eq. (29) is determined up by two constants: the initial amplitude $R_{i, \mathrm{o}}=R_{i}\left(r_{\mathrm{o}}\right)$ and the initial phase $\theta_{i, \mathrm{o}}=\theta_{i}\left(r_{\mathrm{o}}\right)$, where $r_{\mathrm{o}}$ corresponds to the endpoint where the boundary condition is applied. The change of the initial phase $R_{i, \mathrm{o}}$, corresponds to multiply the solution $\mathcal{Y}_{i}$, by a constant factor; thus, the zeros of the wave function $\mathcal{Y}_{i}$ can be located by studying the differential Eq. (A.6). In this sense, this transformation preserves the zeros of the original wave function, and their respective location. The eigenmodes can be determined by the solution of Eq. (A.6) with the convenient boundary conditions. We remember that this analysis is applied to the linear adiabatic non-radial oscillations and consequently the amplitude of waves can be determined up to an arbitrary constant.

We point out that the transformation of the standard differential equation into a self-adjoint form does not conserve necessarily the number of zeros or the location of the original differential equation. The same remains true for all the transformations between equivalent differential equations. However, under the two transformations presented previously to the differential Eq. (4), the selfadjoint transformation and the phase transformation, the number and the location of zeros of the wave function $\Psi$ are preserved. In the case of the self-adjoint form it is necessary to take the convenient formulation, for which the function $\mathcal{M}_{i}$, does not presents zeros in the interval, where the self-adjoint form is applied.

Furthermore, we point out that the number of zeros of a given eigenfunction is not a unique way to classify 
modes (see Sect. 5). In fact, a classification scheme can be determined independent of that and the number of zeros can be used to classify the modes only in some particular cases.

\section{A.2. Local phase properties}

The properties of the eigenfunction and of the correspondent eigenfrequencies can be determined from Eq. (A.6), with the correspondent boundary conditions. It is possible to build a graph for each eigenmode of a given eigenfrequency $\omega$ and degree $l$, where the variation of the phase with the stellar radius is represented. We call this the linear phase diagram (see Fig. 4), and we will return later to this point.

A solution $\mathcal{Y}_{i}(r)$ of the Eq. (29) has a zero at a point $r=r_{1}$ if and only if $\theta_{i}\left(r_{1}\right)=n \pi$, where $n$ is always an integer. At each of these points $\cos \theta_{i}=1$ and $\frac{\mathrm{d} \theta_{i}}{\mathrm{~d} r}=\mathcal{P}_{i}^{-1}(r)$. On the propagation regions where $k_{\mathrm{r}}^{2}>0$, this means geometrically that each curve $\left(\mathcal{P}_{i} \mathcal{Y}_{i}^{\prime}, \mathcal{Y}_{i}\right)$ in the plane $\left(\mathcal{P}_{i} \mathcal{Y}_{i}^{\prime}, \mathcal{Y}_{i}\right)$, corresponding to a solution $\mathcal{Y}_{i}$ of the differential Eq. (29) (for a given eigenvalue $\omega$ ), can cross the $\mathcal{P}_{i} \mathcal{Y}_{i}^{\prime}$ axis $(\theta=n \pi)$ counterclockwise when $\mathcal{P}_{i}>0$, and clockwise when $\mathcal{P}_{i}<0$. The solution $\mathcal{Y}_{i}(r)$ of the Eq. (29) (or equivalently of the Eq. (A.6)) for a given interval can lead to one of two possible cases: If $\operatorname{sgn}\left(\mathcal{P}_{i}(r)\right)=\operatorname{sgn}\left(\mathcal{Q}_{i}(r)\right)$ then it has a discrete number of zeros, if any at all. Alternatively, if $\operatorname{sgn}\left(\mathcal{P}_{i}(r)\right) \neq \operatorname{sgn}\left(\mathcal{Q}_{i}(r)\right)$ then it has either one zero or no zero at all. The waves are propagative only, where $\operatorname{sgn}\left(\mathcal{P}_{i}(r)\right)=\operatorname{sgn}\left(\mathcal{Q}_{i}(r)\right)$ and they are evanescent elsewhere. In the case of the acoustic propagative region $\left(f_{\mathrm{p}}>0\right.$ and $\left.f_{\mathrm{g}}>0\right)$ the phase is increasing with the radius $\left(\frac{\mathrm{d} \theta_{i}}{\mathrm{~d} r}>0\right)$ and in the case of gravity propagative region $\left(f_{\mathrm{p}}<0\right.$ and $\left.f_{\mathrm{g}}<0\right)$ the phase is decreasing with the radius $\left(\frac{\mathrm{d} \theta_{i}}{\mathrm{~d} r}<0\right)$. In case of pure acoustic modes, the phase $\theta_{i}$ is always increasing $\left(\frac{\mathrm{d} \theta_{i}}{\mathrm{~d} r}>0\right)$ in all the interval where the wave is propagative, and decreasing elsewhere. This works conversely for the pure gravity modes.

In resume, we can identify a particular phase signature of modes related with the propagative behavior in a given region. Then locally a gravity type wave and a acoustic type wave, propagates depending upon whether the phase point is traveling clockwise (gravity or g-modes, where $\frac{\mathrm{d} \theta_{i}}{\mathrm{~d} r}>0$ ), or counterclockwise (acoustic or p-modes, where $\frac{\mathrm{d} \theta_{i}}{\mathrm{~d} r}<$ $0)$, at points of $\theta_{i}\left(r_{1}\right)=n \pi$, as $r$ increases. The global behavior of the eigenmodes is discussed in the Sect. 3.2.

\section{Appendix B: Determination of the boundary conditions in the case of planar approximation}

In this section, we present the calculation of the endpoints conditions of the associated phase system, applied to equations of motion for adiabatic oscillations when the wavelength is much less than the solar radius, the local effects of spherical geometry on the dynamic can be ignored (Deubner \& Gough 1984, see also Sect. 2.1.1). To simplify the determination of the endpoints of the phase equations, from the boundary conditions (see Sect. 2.2), we will consider the phase equations, on the approximation proposed in Sect. 3.4 (Eqs. (51) and (52)). Furthermore, we will consider only the dominant terms for $\omega_{+}^{2}$ and $\omega_{-}^{2}$ in the planar approximation, given by Eq. (26), which are proportional to $S_{1}^{2}$ and $N^{2}$, respectively. This example is particularly interesting because the boundary conditions corresponding to that case remain the same for the other approximations by the fact that the dominant terms which determine the local behaviour of the phase are presented in this case. The functions, $f_{\mathrm{p}}$ and $f_{\mathrm{g}}$ are given approximately by

$f_{\mathrm{p}} \approx \frac{\omega}{c}\left(1-\frac{L^{2}}{\omega^{2}} \frac{c^{2}}{r^{2}}\right)$

and

$f_{\mathrm{g}} \approx \frac{\omega}{c}\left(1-\frac{N^{2}}{\omega^{2}}\right)$

The boundary condition, of the $\Psi$ function, can be determined from the boundary conditions of $\delta p$, by the Eq. (6) and the respective boundary conditions for $\delta p$. The regularity condition in the centre for $\Psi$ is

$\frac{\mathrm{d} \ln \Psi}{\mathrm{d} r} \rightarrow \frac{l}{r}$

as $r \rightarrow 0$. The boundary condition on the surface, for $\Psi$ is $\Psi=0$

at $r=R$. Using the phase transformation (Eq. (A.5)),

$\cot \theta_{i}=\operatorname{sgn}\left(\mathcal{M}_{i}\right)\left|\mathcal{M}_{i}\right|^{2}\left[-\frac{\mathrm{d}}{\mathrm{d} r} \ln \left|\mathcal{M}_{i}\right|+\frac{\mathrm{d}}{\mathrm{d} r} \ln |\Psi|\right]$

for each one of the descriptions $i=1,2$. We observe that for the centre, from the series expansions of equilibrium quantities we obtain (as $r \rightarrow 0$ ),

$\frac{\mathrm{d}}{\mathrm{d} r} \ln \left|f_{\mathrm{p}}\right|^{1 / 2} \rightarrow r^{-1}$

and

$\frac{\mathrm{d}}{\mathrm{d} r} \ln \left|f_{\mathrm{g}}\right|^{1 / 2} \rightarrow 0$

Under these considerations, it is possible to determine the initial phase of the associated phase system. The endpoints for the centre are $(r \rightarrow 0)$. In the case of the acoustic description, we obtain

$\cot \theta_{1} \approx \operatorname{sgn}\left(f_{\mathrm{g}}\right) \frac{c_{\mathrm{O}}}{\omega} \frac{1}{1-\frac{N^{2}}{\omega^{2}}} \frac{l}{r} \rightarrow+\infty$

or

$\theta_{1}=0(\bmod \pi)$.

In the case of the gravity description, we obtain

$\cot \theta_{2} \approx \operatorname{sgn}\left(f_{\mathrm{p}}\right) \frac{c_{\mathrm{o}}}{\omega} \frac{r^{2}}{r^{2}-\frac{L^{2}}{\omega^{2}} c_{\mathrm{o}}^{2}} \frac{l}{r} \rightarrow 0$ 
or

$\theta_{2}=\frac{\pi}{2}(\bmod \pi)$

The endpoints for the surface, can also be calculated for both descriptions. It is also possible to determine the amplitude boundary conditions, but they are not interesting to our problem.

At last, it is important to observe that the initial boundary conditions can be understood in terms of the physical nature of the acoustic and gravity waves.

\section{References}

Cowling, T. G. 1941, MNRAS, 101, 367

Cox, J. P. 1980, Theory of stellar pulsation (Princeton University Press)

Deubner, F.-L., \& Gough, D. O. 1984, ARA\&A, 22, 593

Eckart, C. 1960, Hydrodynamics of Oceans and Atmospheres (Pergamon, London)

Gabriel, M., \& Scuflaire, R. 1979, Acta Astron., 29, 135

Gough, D. O. 1990, Progress of Seismology of the Sun and Stars, ed. Y. Osaki, \& H. Shibahashi (Springer-Verlag, Dordrecht), 283

Gough, D. O. 1993, Astrophysical fluid dynamics, ed. J.-P. Zahn, \& J. Zinn-Justin (Elsevier, Amsterdam), 399

Gough, D. O. 1996, The structure of the sun, ed. T. Roca Cortés (Cambridge University Press), 141
Gough, D. O., \& Toomre, J. 1991, ARA\&A, 29, 627

Lamb, H. 1932, Hydrodynamics (Cambridge University Press)

Ledoux, P., \& Walraven, T. 1958, Handbuch der Physik, ed. S. Flügge (Springer, New York), 353

Lee, U. 1985, Publ. Astron. Soc. Japan, 37, 262

Lopes, I. P. 2001, MNRAS, 321, 615

Lopes, I. P. 2000, ApJ, 542, 1071

Lopes, I. P., \& Gough, D. O. 2001, MNRAS, 322, 473

Lopes, I., Turck-Chiéze, S., Michel, E., \& Goupil, M.-J. 1997, ApJ, 480, 794

Osaki, Y. 1975, Publ. Astron. Soc. Japan, 27, 237

Osaki, Y. 1976, Publ. Astron. Soc. Japan, 28, 105

Provost, J., \& Berthomieu, G. 1986, A\&A, 165, 218

Prüfer, H. 1926, Mathematische Annalen, 95

Scuflaire, R. 1974, A\&A, 36, 107

Shibahashi, H. 1979, Publ. Astron. Soc. Japan, 31, 87

Shibahashi, H., \& Osaki, Y. 1976a, Publ. Astron. Soc. Japan, 28, 199

Shibahashi, H., \& Osaki, Y. 1976b, Publ. Astron. Soc. Japan, 28,533

Smeyers, P., \& Tassoul, M. 1988, ApJS, 65, 429

Tassoul, M. 1980, ApJS, 43, 469

Tassoul, M. 1990, ApJ, 358, 313

Unno, W., Osaki, Y., Ando, H., Saio, H., \& Shibahashi, H. 1989, Nonradial Oscillations of Stars (University of Tokyo Press)

Vorontsov, S. V. 1991, Sov. Astron. Lett., 35(4), 400 